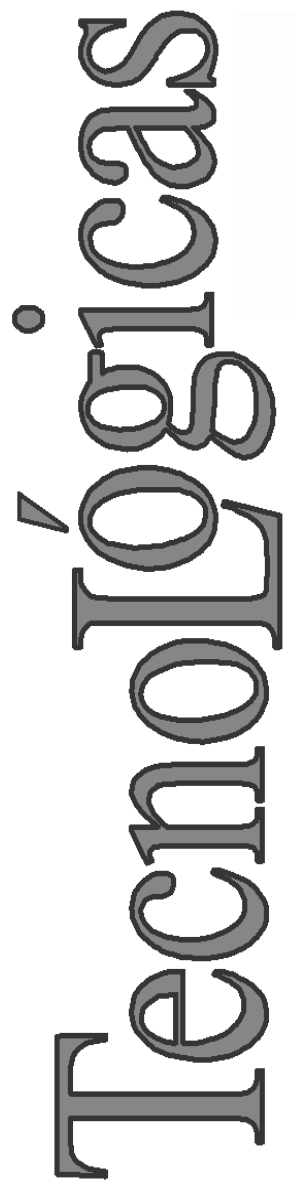

\title{
Sistema de Rehabilitación basado en el Uso de Análisis Biomecánico y Videojuegos mediante el Sensor Kinect
}

\section{Rehabilitation System based on the Use of Biomechanical Analysis and Videogames through the Kinect Sensor}

John E. Muñoz-Cardona ${ }^{1,2,3}$

Oscar A. Henao-Gallo ${ }^{2,3}$ José F. López-Herrera ${ }^{3}$

1 Maestría en Ingeniería Eléctrica, Facultad de Ingenierías, Universidad Tecnológica de Pereira, Pereira-Colombia jemunozc@misena.edu.co

2 Grupo de Investigación y Desarrollo en Cultura de la Salud, Facultad de ciencias de la Salud, Universidad Tecnológica de Pereira, Pereira-Colombia oscarhe@utp.edu.co

3 HCI Group, Unidad de Acción Motora, Clínica de Dolor del Eje Cafetero, Pereira-Colombia jflopez@utp.edu.co 


\title{
Resumen
}

El presente artículo muestra la creación de un novedoso sistema para la rehabilitación física de pacientes con múltiples patologías, a través de dinámicas con videojuegos de ejercicio (exergames) y el análisis de los movimientos de los pacientes usando un software desarrollado. Este sistema está basado en el uso del sensor Kinect para ambos fines: divertir al paciente en su terapia a través de exergames y proporcionarle al especialista una herramienta para el registro y análisis de datos de captura de movimiento (MoCap) tomados a través del sensor Kinect y procesados utilizando análisis biomecánico mediante la transformación angular de Euler. Todo el sistema interactivo se encuentra instalado en un centro de rehabilitación y actualmente se realizan investigaciones con diferentes patologías (stroke, IMOC, trauma craneoencefálico, entre otros), los pacientes realizan sus sesiones con el sistema interactivo mientras el especialista registra los datos para un posterior análisis, el cual se realiza en un software creado para dicho fin. El software arroja gráficas de movimiento en los planos sagital, frontal y rotacional de 20 puntos distribuidos en el cuerpo. El sistema final es portable, no-invasivo, económico, de interacción natural con el paciente y de fácil implementación por parte del personal médico.

\section{Palabras clave}

Kinect, exergame; rehabilitación; análisis biomecánico.

\begin{abstract}
This paper presents development of a novel system for physical rehabilitation of patients with multiple pathologies, through dynamic with exercise videogames (exergames) and analysis of the movements of patients using developed software. This system is based on the use of the Kinect sensor for both purposes: amusing the patient in therapy through of specialist exergames and provide a tool to record and analyze MoCap data taken through the Kinect sensor and processed using biomechanical analysis through Euler angles. All interactive system is installed in a rehabilitation center and works with different pathologies (stroke, IMOC, craneoencephallic trauma, etc.), patients interact with the platform while the specialist records data for later analysis, which is performed by software designed for this purpose. The motion graphics are shown in the sagittal, frontal and rotationalplanefrom 20 points distributed in the body. The final system is portable, non-invasive, inexpensive, natural interaction with the patient and easily implemented for medical purposes.
\end{abstract}

\section{Keywords}

Kinect; exergame; rehabilitation; biomechanical analysis. 


\section{INTRODUCCIÓN}

Dentro del contexto de los juegos serios, existen un tipo específico que busca fomentar la actividad física mediante diferentes roles de juego, los Exergames, estimulan la movilidad del cuerpo entero mediante el uso de ambientes interactivos con experiencias de inmersión que simulan diferentes sensaciones de presencia. Múltiples estudios con personas mayores, profundizando en aspectos de uso y experiencia de usuario propuestos por Exergames de diferentes deportes como golf, boxeo, tenis, voleibol, fútbol, entre otros, han arrojado resultados positivos en la combinación de los elementos del ejercicio físico con el entretenimiento, en donde los adultos mayores en general, tienden a mejorar diferentes actitudes de comportamiento, manteniéndolos activos físicamente y con percepción positiva y graciosa hacia lo juegos (Muñoz et al., 2013b).

Uno de los problemas más comunes para la implementación de sistemas de captura de movimiento es su elevado costo y la complejidad de su montaje. A pesar de la existencia de múltiples sensores de movimiento de bajo costo creados por las industrias de los videojuegos para mejorar las experiencias con sus consolas, cuando son utilizados en el contexto de la rehabilitación física, suelen tener inconvenientes para la captura objetiva de datos de movimiento (posiciones, velocidades, aceleraciones, ángulos) a través de herramientas computacionales. Es por esto que muchos de los estudios que se plantean con el uso de Exergames para la rehabilitación suelen arrojar resultados muy subjetivos y anecdóticos, limitando los alcances de las investigaciones (Kato, 2012).

Ahora, uno de los sensores más renombrados en el campo de los Exergames es el sensor Kinect, el cual permite una perfecta integración con un ordenador más allá de la consola Xbox-360; en rehabilitación, las características de seguimiento y asignación automática de puntos de interacción (Joints) a los usuarios, han convertido a este sensor en una potente herramienta para el análisis objetivo del movimiento (Chang et al., 2012). En este artículo se describe el desarrollo de un novedoso sistema de interacción basado en el uso de éste sensor como herramienta para el análisis biomecánico de pacientes con diferentes tipos de lesiones en un 
centro de rehabilitación en la ciudad de Pereira. La consigna entonces es: mientras el paciente juega activamente un exergame diseñado para fines de rehabilitación específicos, el especialista registra los movimientos del paciente para analizarlos en un software desarrollado para el análisis de datos de captura de movimiento adquiridos mediante el sensor Kinect.

\subsection{Descripción del Sensor Kinect}

El sensor Kinect es una plataforma de videojuegos adaptable al X-Box 360 creado por Microsoft, el cual brinda una experiencia de juego sin controles, en donde los jugadores sin necesidad de usar ningún tipo de periférico, utilizan los movimientos y gestos de su cuerpo, así como su voz para desenvolverse en los diferentes entornos de juego (Fretz, 2011). El Hardware del sensor Kinect es un arreglo de múltiples dispositivos electrónicos: una cámara RGB estándar de 640x480 píxeles de resolución, una cámara de profundidad infrarroja de 320×240 píxeles de resolución, un proyector de láser infrarrojo, un arreglo de micrófonos, y un motor para el movimiento del ángulo de deflexión.

Tanto la cámara de profundidad como la RGB del sistema Kinect pueden capturar datos a una tasa de 30 FPS (cuadros por segundo) a una resolución de $640 \times 480$ píxeles. El sensor permite el seguimiento efectivo de hasta 20 joints distribuidos alrededor del cuerpo según se muestra en la Fig. 1.

\subsection{Sensor Kinect como Herramienta para la Rehabilitación}

El Kinect permite realizar captura de movimiento (MoCap) en un formato estándar BioVisionHierarchical (bvh) (Parent et al., 2010) que puede ser usado posteriormente en un software diseñado para el análisis biomecánico. Se han realizado investigaciones comparativas del Kinect con otros sistemas de captura de movimiento que requieren grandes montajes y costosos equipos (Fernandez et al., 2012); estos trabajos concluyen que si bien el sensor Kinect es menos preciso, las mediciones se encuentran dentro de un rango confiable en aspectos como posiciones y ángulos de movimiento. 

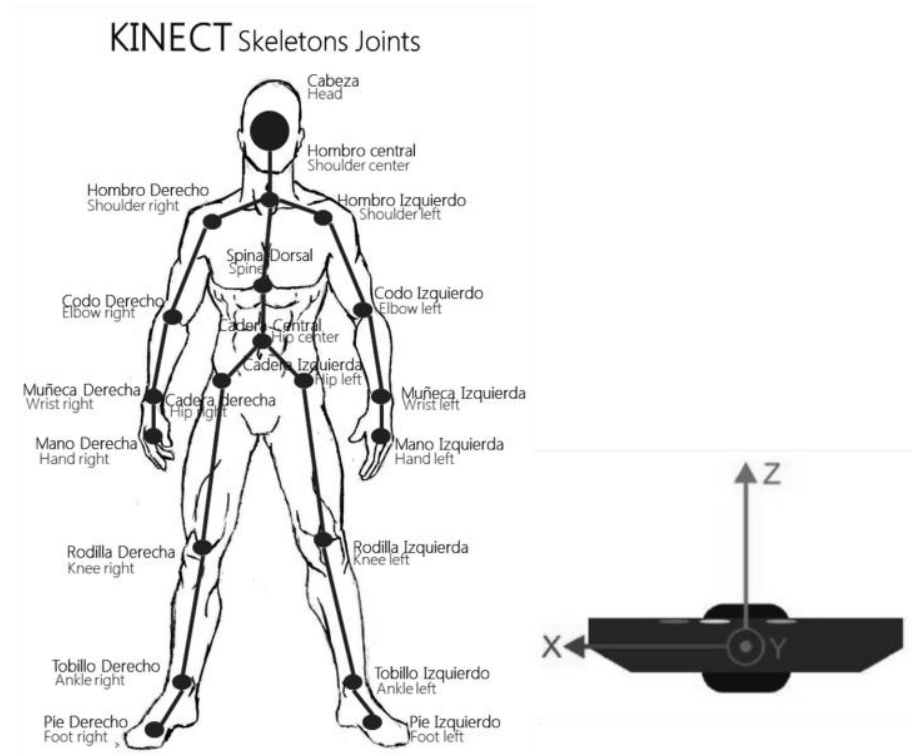

Fig. 1. Izquierda: convención de Joints manejada por el archivo BVH capturado a través del sensor Kinect. Derecha: sistema coordenado del sensor Kinect

Existen también trabajos que apuntan hacía mediciones de balance y equilibrio de los usuarios (Kayama et al., 2012), mientras que otros realizan una extraordinaria combinación entre videojuegos entretenidos y captura objetiva de datos para generar un diagnóstico más asertivo (Chang et al., 2012). El Kinect tiene un rango límite para distancia desde 1,2 m hasta 3,5 m. El área de interacción es alrededor de $6 \mathrm{~m}^{2}$ (Kramer et al, 2012).

Los principales motivos por los cuales el sensor Kinect es considerado como una herramienta exitosa para la rehabilitación son los siguientes: El bajo costo del sensor comparado con el sistema de captura de movimiento más económico del mercado. La portabilidad y comodidad en el uso: los datos de captura pueden ser procesados de manera remota por el especialista para generar un diagnóstico sin necesidad de un desplazamiento físico del paciente hacía un centro especializado, y usando el sensor Kinect no son necesarias condiciones específicas de iluminación. La perfecta integración con herramientas de animación y videojuegos permite 
generar actividades interactivas usando ambientes virtuales. La posibilidad de realizar registro de datos tiempo real de los pacientes convierte al sensor Kinect en una potente herramienta para el análisis biomecánico de las articulaciones.

Plataformas como IGEN de la Universidad del Milano, VirtualRehab de la empresa VirtualWare y SeeMe son claros ejemplos de sistemas robustos para el manejo de terapias de rehabilitación a través del sensor Kinect. Adicionalmente en (Muñoz et al, 2013a) se realiza una combinación de datos de MoCap con datos provenientes de una interface cerebro computador, añadiendo más marcadores biológicos al estudio con videojuegos para la salud.

\subsection{Análisis Biomecánico a través de Ángulos de Euler}

En el cuerpo humano cada segmento está unido a su adyacente formando las articulaciones que son los puntos fijos sobre los que tiene lugar los cambios de posición de los segmentos. Como el movimiento de los cuerpos en el espacio tiene seis grados de libertad, estudiar la dinámica del movimiento del cuerpo humano requiere seis coordenadas independientes. Tres variables corresponden a los centros de masa del miembro del cuerpo, coordenadas XYZ y tres coordenadas de movimiento, o ángulos de Euler. Dados estos sistemas de coordenadas con origen común, es posible especificar la posición de un sistema en términos del otro usando tres ángulos a, 8 y y. Los ángulos de Euler constituyen un medio para dar una descripción numérica de cualquier rotación en un espacio tridimensional usando tres números.

Sin embargo, este abordaje ha sido muy criticado como método de describir los movimientos articulares, ya que el uso de este método impone un orden de secuencia muy estricto en la descripción de las rotaciones que no puede ser alterado (Woltring 1991). Debido a este problema con la descripción goniométrica de los ángulos en 1983 Grood y Suntay introdujeron el concepto de un sistema coordenado de una articulación para describir los ángulos de Euler en las articulaciones del cuerpo humano (Grood\&Suntay, 1983). Para visualizar de manera más fácil los ángulos de Euler para un investigador clínico de biomecánica, ellos determinaron los ángulos empleando un análisis vectorial a partir de un sistema 
de coordenadas articulares de su propia posición pero no con un eje fijo sino con un eje flotante o movible en los planos frontal, sagital y rotacional, describiendo los tres movimientos naturales de las articulaciones del cuerpo humano en coordenadas espaciales que permiten determinar los ángulos de Euler de cada una de ellas de una manera más acertada.

\section{METODOLOGía}

Para el desarrollo del sistema interactivo se tienen en cuenta tres etapas, las cuales describen interactividad, naturalidad y potabilidad de datos para su análisis por parte del fisioterapeuta.

Interacción: la prueba comienza cuando al usuario se le proyecta el videojuego. Los juegos desarrollados son comúnmente de una dinámica sencilla, en donde se busca introducir al paciente en un rol de juego y un nivel de competitividad bastante trivial; agarrar elementos, saltar obstáculos, moverse, inclinarse y balancearse con equilibrio hacen parte activa de la interacción propuesta por estos videojuegos desarrollados. El tiempo de interacción puede ser programado por el especialista según el paciente y las características del videojuego; a menudo el usuario puede utilizar elementos como pesas, bandas y otro tipo de elemento fisioterapéutico que no obstruya ampliamente el espacio entre el usuario y el sensor.

Captura de datos: de forma paralela el especialista debe ejecutar una aplicación para el registro de datos de MoCap que permite llevar los datos del sensor Kinect a un archivo bvh. Esta estandarización de los datos provenientes del seguimiento del esqueleto del usuario ha permitido una integración más dinámica con la comunidad médica en el área de rehabilitación con esta tecnología, ya que posibilita transformar una proceso de medición que suele hacerse de manera análoga a través de goniómetros en un proceso totalmente automatizado, cómodo para el usuario, sin conexiones molestas, portable, de fácil instalación y con mediciones confiables. En (Khoshelham, 2012) muestran que el error aleatorio de medición de profundidad aumenta al aumentar la distancia entre el usuario y el sensor y va desde un pocos milímetros hasta un máximo de $4 \mathrm{~cm}$ en el alcance máximo del sensor. El registro del 
usuario es organizado en forma de bitácora de segmentos de cada una de las sesiones para ser analizadas en cualquier momento por el especialista.

Análisis de datos: en esta etapa, el especialista después de haber realizado la sesión con el paciente y obtenido su correspondiente archivo de MoCap, tendrá que usar el software Bio-Cirac v. 1.0, desarrollado por el equipo de trabajo para cargar el archivo en la interfaz de usuario propuesta. Este software usa las librerías desarrolladas por Neil Lawrence para el uso de archivos de MoCap con extensión bvh y permite un extenso análisis biomecánico por medio de los ángulos de Euler de cada unión (joint).

\section{RESULTADOS Y DISCUSIÓN}

Como resultado de la sinergia entre un grupo de investigadores de ingeniería de la Universidad Tecnológica de Pereira y la Clínica de Dolor del Eje Cafetero, se creó el SIR, Salón Interactivo para la Rehabilitación, el cual es un espacio digital diseñado para brindarle al usuario una experiencia virtual de inmersión; al mismo tiempo, realizar mediciones que pretenden dotar al médico o terapeuta de datos tomados de forma ordenada, periódica y de manera precisa y confiable de los usuarios; brindando las herramientas necesarias para generar diagnósticos más asertivos y en un periodo de tiempo menor comparado con otro tipo de procesos y herramientas de medición. El espacio cuenta con un videoproyector, un ordenador de gama media, un sensor Kinect, un equipo de sonido y herramientas varias para la rehabilitación como pesas, pelotas y bandas.

El ordenador está dotado de un conjunto de videojuegos que permiten la rehabilitación de aspectos como equilibrio, coordinación, flexión, extensión, aducción y abducción de miembros superiores; flexión, abducción y rotación interna de cadera y extensión de rodilla, ente otros. Se desarrolló un procedimiento de medición para las sesiones el cual explica la forma en cómo se deben recolectar los datos y como debe ser la interacción del paciente con el sistema interactivo. Después de cada sesión con los pacientes, el fisioterapeuta debe encargarse de guardar los datos de MoCap en 
forma de bitácora y realizar un registro de datos de la sesión en una ficha digital. El desarrollo del software para el análisis biomecánico a partir del sensor Kinect, Bio-Cirac, permite cargar el archivo de MoCap en un entorno de programación, después de escoger el archivo a analizar, el software arroja un gráfico que relaciona los Joints (cada elemento del esqueleto) con el del usuario en un espacio tridimensional. Para obtener las gráficas de los ángulos de Euler de cada Joint basta con seleccionarlo en la gráfica 3D del esqueleto del usuario. Existen dos tipos de gráficos que realiza el software, el primero es el de los ángulos de Euler el cuál es un gráfico bidimensional que contiene la información relativa con cuadros de movimiento vs los 3 Ángulos de Euler, los cuales son calculados según la convención ZXY. En este gráfico se observa el comportamiento del ángulo de precesión (frontal), el ángulo de nutación (sagital) y ángulo de rotación vs el tiempo de captura total en fotogramas (ver Fig. 2).

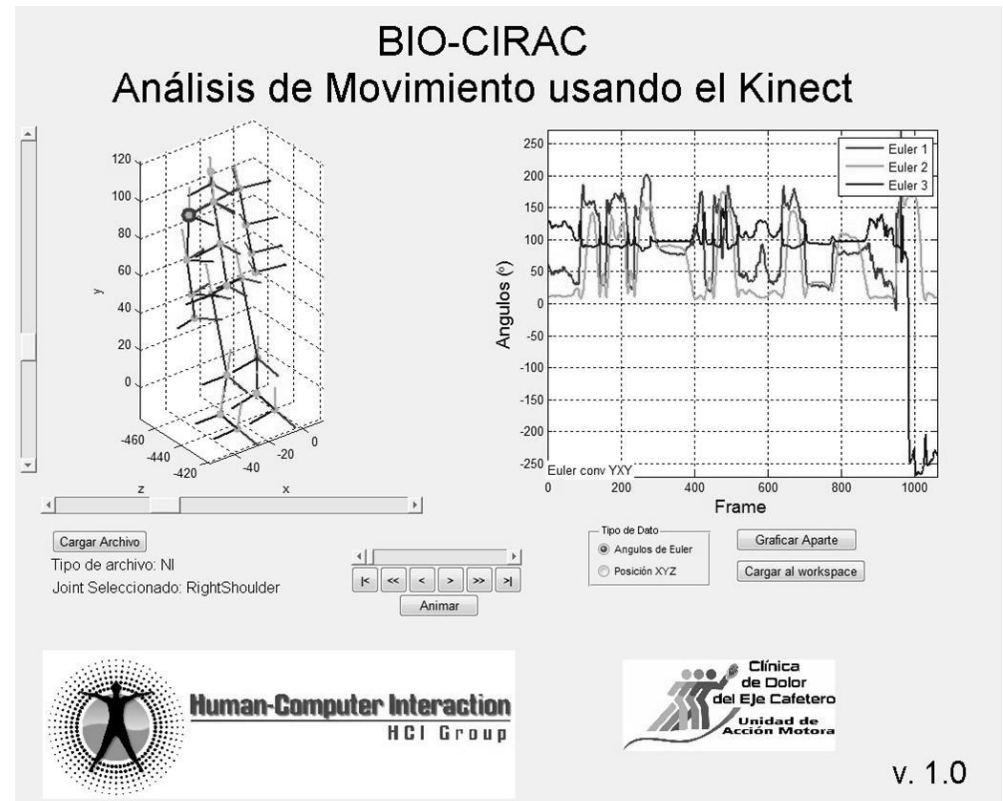

Fig. 2. GUI diseñada para el software Bio-Cirac v. 1.0, la cual permite cargar el archivo de captura de movimiento y generar las gráficas para su análisis 
El segundo tipo de gráfico es el relativo a la posición en XYZ de cada joint, estos datos son capturados de acuerdo al sistema coordenado del sensor Kinect aunque el origen es relocalizado a la posición inicial del cuerpo (en línea con la columna vertebral pero al nivel del piso). El salón interactivo se encuentra en la Unidad de Acción Motora, desde donde se realizan terapias y sesiones de rehabilitación, así como procedimientos de valoración clínica. Este novedoso sistema ya cuenta con publicaciones nacionales e internacionales en donde se refleja el alto nivel de desarrollo tecnológico que encierran estas metodologías (Muñoz, 2013a). Actualmente se desarrollan dos estudios con pacientes con síndrome piramidal y personas sanas titulados "Rehabilitación de la flexión del hombro en pacientes con monoparesia usando un Exergame" y "Valoración del equilibrio estático en personas sanas utilizando un Exergame".

El análisis de los datos de captura de movimiento del estudio de rehabilitación tomados mientras el usuario jugaba, arrojó una mejoría hasta del 18,1 \% en la flexión del hombro en uno de los pacientes con ACV monoparético del estudio. La Tabla 1 muestra un resumen de los aspectos más relevantes de los estudios que se están llevando a cabo en la Clínica de Dolor, en donde este sistema interactivo está siendo evaluado.

Tabla 1. Resumen de los estudios actuales que se desarrollan en la Clínica de Dolor con el sistema interactivo

\begin{tabular}{cccc}
\hline Tipo de Estudio & Aspecto Evaluado & $\begin{array}{c}\text { Cantidad de } \\
\text { Participantes }\end{array}$ & $\begin{array}{c}\text { Resultados } \\
\text { Previos }\end{array}$ \\
\hline $\begin{array}{c}\text { Rehabilitación } \\
\text { con Exergame }\end{array}$ & $\begin{array}{c}\text { Flexo-Extensión del hombro } \\
\text { hemiparético en pacientes } \\
\text { con síndrome piramidal }\end{array}$ & 6 & $\begin{array}{c}\text { Mejora en el } \\
\text { arco de movi- } \\
\text { miento de 18,1\% } \\
\text { en uno de los } \\
\text { pacientes }\end{array}$ \\
$\begin{array}{c}\text { Evaluación con } \\
\text { Exergame }\end{array}$ & $\begin{array}{c}\text { Evaluación del equilibrio } \\
\text { estático en personas sanas }\end{array}$ & 20 & $\begin{array}{c}\text { Mejora en los } \\
\text { tiempos de } \\
\text { equilibrio con } \\
\text { ambos pies para } \\
\text { ambos géneros }\end{array}$ \\
\hline
\end{tabular}

La implementación de técnicas de realidad virtual con captura de movimiento a través de sensores de bajo costo, presupone una 
oportunidad real para la integración de nuevas terapias digitales en rehabilitación física. La medición objetiva de variables espaciales y angulares se presenta como una de las fortalezas que presenta el uso de Exergames en terapias de rehabilitación física.

\section{CONCLUSIONES}

El uso de videojuegos para la salud, específicamente Exergames en un centro de rehabilitación física resulta una metodología complementaria a los procesos de rehabilitación convencionales, añadiendo aspectos de motivación, entretenimiento y distracción de dolor en pacientes con múltiples patologías.

El uso del sensor Kinect como herramienta para el análisis biomecánico del movimiento humano se presenta como un instrumento de medición para variables angulares y de posicionamiento en tres dimensiones, que facilita y optimiza la captura y el análisis de los datos del movimiento los pacientes, dentro de un rango confiable de medida. La convergencia entre el sector académico ingenieril y la empresa privada en salud, establecen un marco de partida para futuras innovaciones en el área de la bioingeniería de la región; en donde se busca innovar no a partir de la oferta de productos y servicios de los grupos de investigación, sino a través de la demanda del sector.

\section{AGRADECIMIENTOS}

Este trabajo ha sido posible gracias al trabajo académico realizado por el semillero de Investigación en HCI y la Clínica de Dolor del Eje Cafetero.

\section{REFERENCIAS}

Chang, C-Y, Lange, B., Zhang M., Koenig S., Requejo P., (2012). Towards pervasive physical rehabilitation using Microsoft Kinect.6th Int. Conf. Perv. Comp. tech. health, 159-162. 
Fernández-Baena A., Susín A., Lligadas X., (2012). Biomechanical validation of upper-body and lower-body joint movements of Kinect motion capture data for rehabilitation treatments. ConfINCoS, IEEE, 656661.

Fretz, M., (2011). VENTANA, Interactive shop window.Master thesis, Univ. of arts Zurich, Sping.

Grood E.S, Suntay W.J., (1983). A joint coordinate system for the clinical description of three dimensional motions: Application to the knee. Journal Biomech Eng., 105(2), 136-44.

Kato P. M.: Evaluating eficacy and validating games for health. G4H Journal, February, 1(1): 74-76. doi:10.1089/g4h.2012.1017.

Kayama, H., Kazuya.,Nishiguchi, S., Nagai, K., Yamada, M., (2012). Concept software based don Kinect for assesing dual-task ability of elderly people. G4H Journal, 1(5).

Khoshelham, K., Elberink, S. O., (2012). Accuracy and resolution of Kinect depth data for indoor mapping applications. InTech Op Scien: Sens, 1437-1454.

Kramer, J., Burrus, N., Echtler, F., Herrera, D., Parker, M., (2012). Hacking the Kinect. Book, Technology in action.

Muñoz, J., Henao O., Lopez J., (2013a). BCI Games With Motion Capture and its Possibilities in Rehabilitation. Proce.of the Fifth Int.BCI Meet., Art Id, 055.

Muñoz, J., Villada J., Giraldo J., (2013b). Exergames: una herramienta tecnológica para la actividad física. Rev Med de Ris, 19(2), 126-130.

Parent, R., Ebert, D.S., Gould, D., (2010). Computer animation complete. All-in-one: learn motion capture characteristic, point-based, and maya winning techniques. Elsevier's Sci\& Tech Rights Dept, 113-117.

Woltring, H., (1991). Representation and calculation of 3-D joint movement. Hum Move Sci, 10(5), 603-616. 